Artículo científico

Volumen 30(2):407-424. Mayo-agosto, 2019

e-ISSN 2215-3608, doi:10.15517/am.v30i2.33997

https://revistas.ucr.ac.cr/index.php/agromeso/index

\title{
Absorción de nutrientes en arroz en un suelo inceptisol bajo riego en Coclé, Panamá ${ }^{1}$
}

\section{Absorption of nutrients in rice in an inceptisol soil under irrigation in Coclé, Panamá}

\author{
Luis Alberto Barahona-Amores², José Ezequiel Villarreal-Núñez², Walker González-Carrasco², \\ Evelyn Itzel Quiro-Mclntire ${ }^{2}$
}

1 Recepción: 16 de julio, 2018. Aprobación: 12 de diciembre, 2018. Este trabajo formó parte de la investigación "Curvas de absorción de nutrientes en diferentes tipos de suelo y ambiente agroecológico dedicados al cultivo de arroz" financiada con fondos del proyecto para mejorar la competitividad de los sistemas productivos de arroz en Panamá. Instituto De Investigación Agropecuaria de Panamá (IDIAP), Panamá.

2 Instituto De Investigación Agropecuaria de Panamá (IDIAP), Panamá. alberline@gmail.com (https://orcid.org/0000-0001-5824-7688), jevilla38@gmail.com,walkerdcg@gmail.com, evelynitzel26@gmail.com

\section{Resumen}

Introducción. El contenido de nutrientes en la planta de arroz (Oryza sativa) durante sus etapas fenológicas del cultivo es afectado por la disponibilidad de nutrientes en el suelo, la dosis y fuente de los fertilizantes, las variedades y los sistemas de cultivos utilizados. Objetivo. El objetivo de este trabajo fue determinar la extracción de macro y micro nutrientes en dos variedades de arroz según etapa fenológica, en un suelo Inceptisol bajo condiciones de riego. Materiales y métodos. El estudio se realizó en Penonomé, Coclé, Panamá, en los ciclos agrícolas del 2015 y 2016. Se utilizaron las variedades de arroz IDIAP FL 106-11 e IDIAP FL 137-11. Se realizaron muestreos de plantas cada quince días a partir de la germinación con cuatro réplicas y empleo de un marco de 0,25 m x 0,25 m. Se realizó un análisis de regresión para determinar la extracción de nutrientes por etapa de cultivo. Resultados. La variedad IDIAP FL 106-11 produjo 31,7 t.ha-1 de materia seca y la IDIAP FL 137-11 26,9 t.ha-1; ${ }^{-1}$ la producción de grano fue similar con

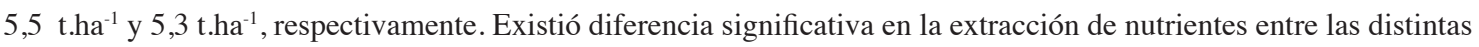
etapas fenológicas en ambas variedades (p<0,001). IDIAP FL 106-11 extrajo mayor cantidad de $\mathrm{N}, \mathrm{K}, \mathrm{Mn}, \mathrm{Zn}$ y $\mathrm{Cu}$ que IDIAP Fl 137-11, la cual absorbió mayor cantidad de P, Ca, Mg y Fe. El orden de importancia de los nutrientes basados en la cantidad extraída fue $\mathrm{K}>\mathrm{N}>\mathrm{Ca}>\mathrm{P}>\mathrm{Mg}>\mathrm{Mn}>\mathrm{Fe}>\mathrm{Zn}>\mathrm{Cu}$. Bajo las condiciones del estudio IDIAP FL 106-11 exigió mayor cantidad de nutrientes para producir una tonelada de grano que IDIAP FL 137-11. Conclusión. Cada variedad presentó distinta absorción de nutrientes lo que sugiere un ajuste en los planes de fertilización que se manejan en la actualidad.

Palabras claves: absorción, fenología, fertilización, nutrientes, variedades de arroz.

\begin{abstract}
Introduction. Nutrients content in rice (Oryza sativa) plant during its different phenological stages is affected by nutrients content in soil, dose and fertilizers source, varieties, and implemented cropping systems. Objective. The objective of this research was to determine the extraction of macro and micro nutrients from two rice varieties in each phenological stage, in an Inceptisol soil under irrigated conditions. Materials and methods. The study was developed in Penonome, Cocle, Panama in the 2015 and 2016 agricultural cycles. The IDIAP FL 106-11 and IDIAP FL 137-11 varieties were used. Plants samples were taken every fifteen days since germination with four repetitions
\end{abstract}


using a frame of $0.25 \mathrm{~m} \mathrm{X} 0.25 \mathrm{~m}$. A regression analysis was performed to determine the nutrients extraction per crop stage. Results. IDIAP FL 106-11 variety yielded 31.7 t.ha ${ }^{-1}$ of dry matter and the IDIAP FL 137-11 about 26.9 t.ha ${ }^{-1}$; grain yield was similar with $5.5 \mathrm{t}^{-h^{-1}}$ and $5.3 \mathrm{t}^{\mathrm{h} \mathrm{h}^{-1}}$, respectively. There were significant differences about nutrients extraction between the different phenological stages in both varieties $(\mathrm{p}<0.001)$. IDIAP FL 106-11 extracts more $\mathrm{N}$, K, $\mathrm{Mn}, \mathrm{Zn}$, and $\mathrm{Cu}$ than IDIAP FL 137-11, which absorbed more P, Ca, Mg, and Fe. Nutrients importance order, based on extracted quantity, was $\mathrm{K}>\mathrm{N}>\mathrm{Ca}>\mathrm{P}>\mathrm{Mg}>\mathrm{Mn}>\mathrm{Fe}>\mathrm{Zn}>\mathrm{Cu}$. In a study conditions, IDIAP FL 106-11 requires greater amounts of nutrients to produce a ton of grain than IDIAP FL 137-11. Conclusion. Each variety presented nutrient different absorption implying an adjustment in the fertilization plans that are currently handled.

Keywords: absorption, phenology, fertilization nutrients, rice varieties.

\section{Introducción}

En el cultivo del arroz (Oryza sativa), la fertilización constituye un factor importante en la obtención de alta productividad. La respuesta a la fertilización depende de la variedad, la fertilidad del suelo, el clima, el manejo del agua y del control de plagas y enfermedades (INPOFOS, 2004; Molina y Rodríguez, 2012). Con base en el consumo total de nutrientes de un cultivo, se puede estimar la dosis de nutrientes necesarios para obtener una productividad dada. Esto se logra confrontando el consumo total con las cantidades presentes en el suelo, para determinar las cantidades de nutrientes necesarios para llegar a la meta de productvidad deseada (Ramírez y Bertsch, 2002).

Los elementos minerales necesarios para el desarrollo y metabolismo de las plantas se denominan nutrientes. Son elementos esenciales que desempeñan funciones específicas dentro de la planta, la ausencia de ellos impiden el desarrollo del ciclo biológico, y ningún otro elemento puede sustituirle. Los elementos nutritivos esenciales para el arroz son carbono $(\mathrm{C})$, oxígeno $(\mathrm{O})$, hidrógeno $(\mathrm{H})$, nitrógeno $(\mathrm{N})$, fósforo $(\mathrm{P})$, potasio $(\mathrm{K})$, calcio $(\mathrm{Ca})$, magnesio $(\mathrm{Mg})$, azufre $(\mathrm{S})$, hierro $(\mathrm{Fe})$, manganeso $(\mathrm{Mn})$, molibdeno $(\mathrm{Mo})$, cobre $(\mathrm{Cu})$, boro $(\mathrm{B})$, zinc $(\mathrm{Zn})$ y cloro $(\mathrm{Cl}), \mathrm{y}$ silicio ( $\mathrm{Si}$ ), este último incluido por estudios recientes (Rodríguez y Fórez, 2004).

El $\mathrm{N}$ es un regulador del crecimiento que promueve la reproducción celular y, por ende, el macollamiento de la planta (Mejía y Menjívar, 2010). De igual manera existe una correlación positiva entre el número de granos por unidad de área y el porcentaje de $\mathrm{N}$ absorbido por la planta momentos antes de la floración (Yoshida, 1978).

A diferencia de lo que ocurre con el $\mathrm{N}$, los iones fosfato no se reducen químicamente dentro de la planta, donde la mayor parte del fosfato absorbido se incorpora a moléculas orgánicas (como los ésteres), y estos compuestos se acumulan en las raíces y en las hojas hasta la iniciación de la panícula (Perdomo et al., 1985).

El K es de gran importancia en el desarrollo de las macollas de la planta, en la formación de la panícula y en la resistencia al acame. El Ca funciona como segundo mensajero en la conducción de señales entre los factores ambientales y los mecanismos de respuesta de las plantas, en términos de crecimiento y desarrollo; esta función se relaciona con la división celular, por tanto, el arroz toma el Ca de manera continua hasta la etapa de llenado de grano. La principal función del Mg está en la fotosíntesis, porque es el átomo central de la molécula de clorofila y se requiere en mayor cantidad en las etapas donde hay mayor producción de biomasa (Mejía y Menjívar, 2010).

Se ha demostrado que en sitios específicos de síntesis proteica de los órganos reproductivos, hay una necesidad grande de Zn (Marschner, 1995). La función principal del Fe es la activación de enzimas, donde actúa como grupo prostético, interviene, por tanto, en las reacciones fundamentales de la diferenciación floral. La falta de $\mathrm{Cu}$ disminuye la formación de espigas o panículas en algunos cereales, lo que indica su importancia en el inicio de la etapa reproductiva (Mejía y Menjívar, 2010).

La importancia de determinar épocas de aplicación de fertilizantes de acuerdo con etapas de máxima absorción, así como la cantidad total de nutrientes que la planta requiere para su desarrollo, radica en que ayudan a desarrollar prácticas adecuadas para mejorar el crecimiento integral de la planta, mediante la aplicación de los nutrientes en 
proporciones adecuadas y optimizar la producción sin degradar los recursos naturales (Magnifico et al., 1979; Baligar y Fageira, 2007; Herber y Kraemer, 2010).

Una curva de absorción de nutrientes es la representación gráfica de la extracción de un nutriente e indica las cantidades de este elemento extraídas por la planta durante su ciclo de vida (Sancho, 1999). Un estudio de absorción es un buen punto de partida para estimar la cantidad de nutrientes que se debe reponer al campo para mantener la fertilidad. Si se desea mantener la sostenibilidad del sistema, se debe al menos reponer al suelo las mismas cantidades de nutrientes que salen directamente del campo en la biomasa del cultivo (Ramírez et al., 2000; Bertsch, 2005).

Las principales etapas fenológicas del cultivo de arroz en las fases vegetativas, reproductiva y maduración fueron descritas por Jarma et al. (2010). La fase vegetativa comprende la germinación de la semilla, macollamiento y termina en la iniciación de la panícula. La fase reproductiva que empieza en la iniciación de la panícula y termina en el inicio de la floración. La fase de madurez fisiológica empieza en el inicio de la floración y termina en la madurez fisiológica del grano.

En la actualidad, los planes de fertilización en Panamá comprenden el uso de fórmulas compuestas de fertilizantes como 12-24-12, 15-30-8 y 10-30-10, donde se aplican a la siembra, con un fraccionamiento del nitrógeno en forma de urea $(46 \% \mathrm{~N})$ a los 30 y 60 días después de siembra, independientemente del ciclo de la variedad. No se utiliza asufre ni micronutrientes (Camargo et al., 2014)

Se han realizado varios trabajos sobre curvas de absorción de nutrientes en diversas variedades de arroz en Panamá (Name y Villarreal, 2004; Villarreal-Barrera, 2018), Argentina (Herber y Kraemer, 2010), Costa Rica (Bertsch, 2005; Molina y Rodríguez, 2012) y Nicaragua (Amador y Bernal, 2012), presentando diferentes extracciones de nutrientes según la variedad, el manejo y el tipo de suelo.

El presente trabajo se realizó con el objetivo de determinar la absorción y extracción de macro y micro nutrientes en dos variedades de arroz según la etapa fenológica, en un suelo inceptisol bajo condiciones de riego.

\section{Materiales y métodos}

\section{Localización geográfica}

El estudio se realizó en el Campo Experimental del Sub-Centro Pacífico Marciaga, en el corregimiento de El Coco, distrito de Penonomé, provincia de Coclé, perteneciente al Instituto de Investigación Agropecuaria de Panamá (IDIAP). Es un área de llanuras que corresponde al bosque seco tropical (Holdridge, 1967), ubicado en 8²5'00" $\mathrm{N} \mathrm{y} 80^{\circ} 21^{\prime} 10^{\prime \prime} \mathrm{O}$ (Figura 1). El suelo es fino, mezclado de textura franco arcillo arenosa, clasificado como Aeric Tropaquept, con un régimen de humedad ústico y un régimen de temperatura isohipertérmico (Jaramillo, 1991).

\section{Material genético}

El material genético utilizado en el experimento fueron las variedades del IDIAP denominadas IDIAP FL 10611 y la IDIAP FL 137-11 (Cuadro 1). Tipo de grano largo y delgado, subespecie índica.

\section{Características edafoclimáticas}

Se realizó un muestreo del suelo antes de la siembra del experimento, a una profundidad de $30 \mathrm{~cm}$ (capa arable), con base en submuestras, las cuales se mezclaron para sacar una muestra homogénea y representativa en 


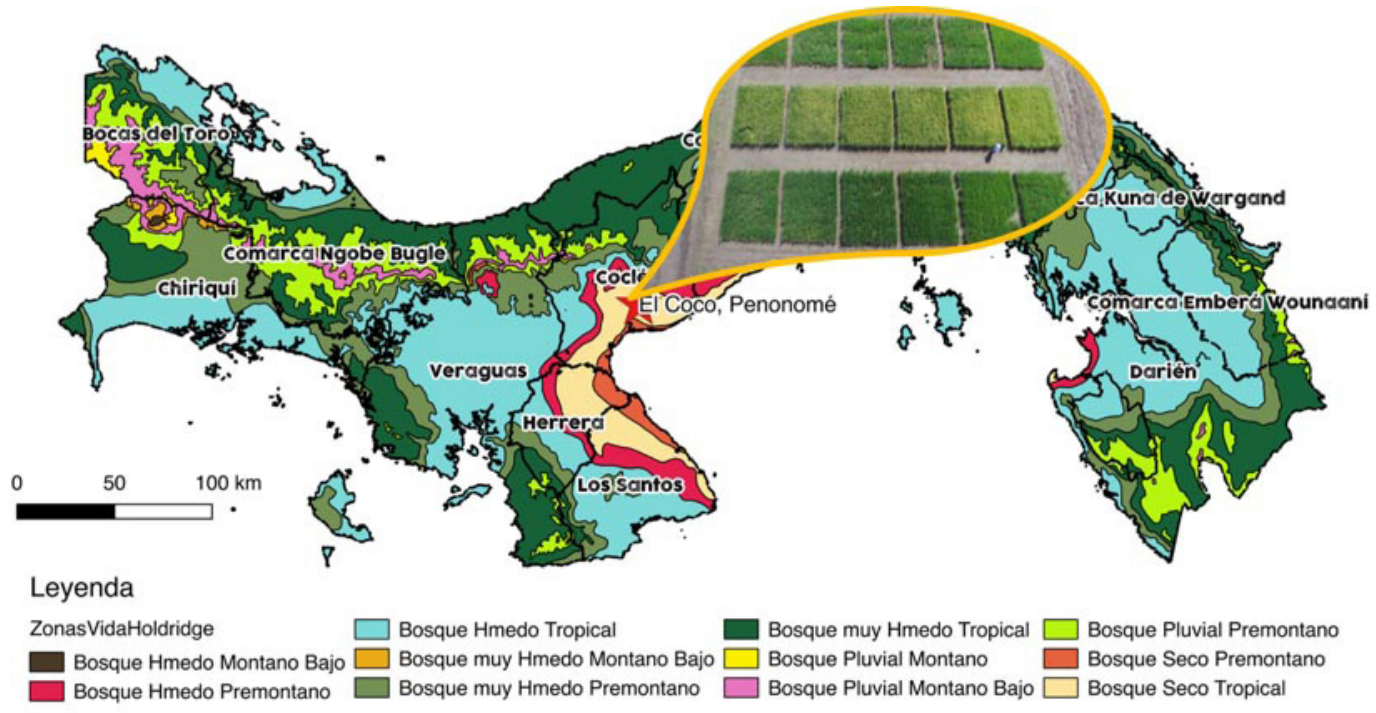

Figura 1. Localización geográfica del estudio de absorción de nutrientes en dos variedades de arroz, de acuerdo con la clasificación de zonas de vida de Holdridge (1967). Campo Experimental Pacífico Marciaga, Penonomé, Panamá. 2016.

Figure 1. Geographic location of the study of the nutrients' absorption in two rice varieties, according to the classification of life zones of Holdridge (1967). Pacifico Marciaga Experimental Field. Penonome, Panama. 2016.

Cuadro 1. Características de dos variedades de arroz utilizadas en el estudio de absorción de nutrientes (Fuente: adaptado de QuirósMclntire et al., 2011).

Table 1. Characteristics of two rice varieties used in the nutrient absorption study (Source: Adapted from Quirós-Mclntire et al., 2011).

\begin{tabular}{lccccc}
\hline \multirow{2}{*}{ Variedad } & MM & IP & Flor & Mat & Prod \\
\cline { 2 - 6 } & & Días después de siembra & & kg/ha \\
\hline IDIAP FL 106-11 & 45 & 53 & 84 & 115 & 5083 \\
IDIAP FL 137-11 & 47 & 57 & 87 & 118 & 5122 \\
\hline
\end{tabular}

$\mathrm{MM}=$ máximo macollamiento, $\mathrm{IP}=$ inicio de primordio, $\mathrm{Flor}=$ floración, $\mathrm{Mat}=$ maduración, Prod= productividad $/ \mathrm{MM}=\operatorname{maximum}$ tillering, IP = beginning of primordium, Flor = flowering, Mat = maturation, Prod $=$ yield.

cada localidad, para determinar sus características fisicoquímicas según la metodología descrita por Villarreal y Name (1996).

En cuanto a contenido de nutrientes y según los niveles críticos establecidos por el Laboratorio de Suelos del IDIAP (Name y Cordero, 1987), se registraron niveles altos de Mn, niveles medios de K y Mg; además, hubo niveles bajos de $\mathrm{P}, \mathrm{Ca}, \mathrm{Al}, \mathrm{Fe}, \mathrm{Zn}$ y $\mathrm{Cu}$. En cuanto a las características físico-químicas del suelo, presentó una textura franco arenosa, con una coloración en seco pardo amarillento (10YR5/8), pH muy ácido, una capacidad de intercambio catiónico efectiva (CICE) baja, con una saturación de Al baja $(5,4 \%)$ y una saturación de bases alta, la cual estaba dominada por la saturación de Ca $(55,7 \%)$ y $\mathrm{Mg}(33,5 \%)$; además de un nivel de materia orgánica bajo (Cuadro 2). 
Cuadro 2. Características físico-químicas del suelo utilizado en el estudio de absorción de nutrientes en arroz. Campo Experimental Pacífico Marciaga, Penonomé, Panamá. 2016.

Table 2. Physico-chemical characteristics of the soil used in the study of nutrient absorption in rice. Pacifico Marciaga Experimental Field, Penonome, Panama. 2016.

\begin{tabular}{|c|c|c|c|c|c|c|c|c|}
\hline $\mathbf{P}$ & $\mathbf{K}$ & $\mathrm{Ca}$ & $\mathbf{M g}$ & Al & Mn & $\mathrm{Fe}$ & $\mathbf{Z n}$ & $\mathrm{Cu}$ \\
\hline \multicolumn{2}{|c|}{$\left(\mathrm{mg} .1^{-1}\right)$} & \multicolumn{3}{|c|}{$\mathrm{cmol}_{(+)} \cdot \mathrm{kg}^{-1}$} & \multicolumn{4}{|c|}{$\left(\mathrm{mg} . \mathrm{l}^{-1}\right)$} \\
\hline 4,0 & 77,0 & 2,1 & 1,2 & 0,2 & 55 & 22 & 0,5 & 1,0 \\
\hline Textura & color & $\begin{array}{c}\text { CICE } \\
\text { cmol }_{(+)} \cdot \mathbf{k g}^{-1}\end{array}$ & pH & & & $\begin{array}{c}\text { bases \% } \\
\text { saturación }\end{array}$ & & \\
\hline FA & 10YR5/8 & 3,7 & 4,9 & & & 94,6 & & \\
\hline
\end{tabular}

\section{Establecimiento y manejo del estudio}

El ensayo se realizó en los ciclos agrícolas del 2015 y 2016, de septiembre a diciembre, con un sistema de riego suplementario. Se utilizaron parcelas de $100 \mathrm{~m}^{2}$ para cada variedad $(10 \mathrm{~m}$ x $10 \mathrm{~m})$; la siembra se realizó sobre suelo seco en hileras separadas cada 0,2 m, con una densidad de siembra de $130 \mathrm{~kg} \cdot \mathrm{ha}^{-1}$ de semilla. Se utilizó una fertilización básica de $120 \mathrm{~kg} \mathrm{~N} \cdot \mathrm{ha}^{-1}, 80 \mathrm{~kg} \mathrm{P}_{2} \mathrm{O}_{5} \cdot \mathrm{ha}^{-1}$ y $80 \mathrm{~kg} \mathrm{~K}_{2} \mathrm{O} \cdot \mathrm{ha}^{-1}$, en donde el total del P y K se aplicó al momento de la siembra con base en la fórmula compuesta de 12-24-12 y el $\mathrm{N}$ se fraccionó en tres partes iguales a los 30, 45 y 55 días después de la siembra (urea $46 \%$ ), con base en los planes de fertilización actual para el cultivo, donde no se utiliza S ni micronutrientes (Camargo et al., 2014).

El manejo agronómico se realizó de acuerdo con las tecnologías generadas por el IDIAP (Camargo et al., 2014). La cosecha se realizó cuando ambas variedades alcanzaron la madurez fisiológica con una humedad de grano de $25 \%$, se limpió y secó para luego determinar la productividad de grano limpio y seco al $14 \%$ humedad.

\section{Muestreo en campo}

Los muestreos de campo para la elaboración de las curvas de absorción se realizaron con base en la metodología descrita por Ramírez y Bertsch (1998), Sancho (1999) y Bertsch (2009). Se muestreó cada quince días, y se aseguró que coincidieran con las etapas fenológicas más importantes del cultivo de arroz. En cada etapa de muestreo se hicieron cuatro réplicas, en cada réplica se utilizó un marco de $0,25 \mathrm{~m}$ x $0,25 \mathrm{~m}$ por $30 \mathrm{~cm}$ de profundidad del suelo. Luego del lavado de raices, las muestras se dividieron en raíz, follaje y granos, para determinar la extracción de nutrientes en cada una de ellas.

Se pesaron las muestras húmedas, luego se llevaron al horno a $70{ }^{\circ} \mathrm{C}$ por $48 \mathrm{~h}$ y se tomó el peso seco para calcular la producción de biomasa seca total $\left(\mathrm{kg} \cdot \mathrm{ha}^{-1}\right)$ por hectárea. Para la producción de grano se utilizó un marco de $1 \mathrm{~m}^{2}$ y se calculó la productividad de grano limpio y seco al $14 \%$ de humedad.

\section{Análisis de laboratorio}

El contenido de nutrientes se determinó según la metodología descrita por Villarreal y Name (1996) y Wilcox y Rivera (2010). Las muestras secas se molieron y pasaron por un tamiz No. 20 (1 mm de abertura en la malla), luego se guardaron en sobres de papel a temperatura ambiente.

El $\mathrm{N}$ se determinó mediante el método Kjeldahl. Se utilizó el proceso de mineralización vía húmeda y se determinó el P por método fosfomolíbdico, con el empleo de un espectrofotómetro de luz visible, y el K, Ca, Mg $\mathrm{Mn}, \mathrm{Fe}, \mathrm{Cu}$ y $\mathrm{Zn}$ se leyeron en absorción atómica de fuente continua. 


\section{Análisis estadístico}

Se realizó una prueba de t-Student (alfa: 0,05) con el software InfoStat versión 18 profesional, para determinar si existieron diferencias entre las variedades en cuanto a la productividad y absorción de nutrientes, además se hizo un análisis de regresión con base en los modelos potencial $\left(y=a^{*} x^{b}\right)$, lineal $(y=a+b x)$, exponencial $\left(y=a b^{x}\right)$ y cuadrática $\left(y=a+b x+c x^{2}\right)$, buscando el que mejor se ajuste para cada nutriente, que permita determinar la absorción de cada elemento en cualquier etapa fenológica del cultivo.

Donde:

$\mathrm{y}=$ absorción de nutriente.

$\mathrm{a}=$ pendiente de la curva.

$\mathrm{b}=$ intercepto de la curva.

$\mathrm{X}=$ variable independiente (etapa de muestreo).

El R ${ }^{2}$ mide la proporción de la variabilidad en los datos de la variable dependiente o Y (absorción de nutrientes), con respecto a la variable independiente o X (fenología del cultivo o días de ciclo), que se explica por el modelo de regresión (Gutierrez y De-La-Vara, 2013).

\section{Variables de estudio}

Las variables evaluadas fueron: contenido de macro y micro nutrientes en el suelo, absorción de nutrientes por etapa fenológica por ha (raíz, follaje y panícula), absorción de nutrientes total por ha (raíz, follaje y panícula), absorción total de nutrientes por ha (raíz+follaje+panícula) y productividad de grano al 14 \% humedad.

\section{Resultados}

\section{Rendimiento y absorción total}

No hubo diferencia en productividad de grano entre ambas variedades $(\mathrm{p}=0,190)$, mientras que para los nutrientes hubo diferencias significativas en la absorción por las dos variedades $(\mathrm{p}<0,001)$.

La variedad IDIAP FL 137-11 absorbió mayor cantidad de P, Ca, Mg y Fe que la variedad IDIAP FL 106-11, mientras esta última absorbió mayor cantidad de N, K, Mn, Zn y Cu (Cuadro 3).

El análisis de regresión mostró que existieron diferencias altamente significativas en la extracción de nutrientes entre las distintas etapas fenológicas del cultivo para las dos variedades $(p<0,001)$. Los coeficientes de determinación o $\mathrm{R}^{2}$ del modelo de regresión, presentaron ajustes entre 67 y $98 \%$. Se determinaron las ecuaciones de regresión para la absorción de nutrientes en ambas variedades (Cuadro 4).

La curva de crecimiento con base en la producción de biomasa seca para ambas variedades tuvo un comportamiento similar, y presentó un aumento lineal hasta la etapa de floración (noventa días después de germinado), donde la variedad IDIAP FL 106-11 continuó incrementando la producción de biomasa, y la IDIAP 137-11 tuvo un decrecimiento. Ambas variedades presentaron productividades estadísticamente similares (Figura 2).

\section{Curva de absorción de nitrógeno}

La absorción de $\mathrm{N}$ por etapa fenológica, tuvo un comportamiento distinto en las dos variedades de arroz $(\mathrm{p}<0,001)$, mientras que la IDIAP FL 106-11 presentó su máxima absorción entre los 75 a 90 días después de 
Cuadro 3. Absorción total de nutrientes por hectárea y extracción por tonelada de grano producido, en dos variedades de arroz. Campo Experimental Pacífico Marciaga, Penonomé, Panamá. 2016.

Table 3. Total absorption of nutrients per hectare and extraction per ton of grain produced in two varieties of rice. Pacifico Marciaga Experimental Field, Penonome, Panama. 2016.

\begin{tabular}{|c|c|c|c|c|c|c|c|c|c|}
\hline \multirow[t]{2}{*}{ Variedad } & $\mathbf{N}$ & $\mathbf{P}$ & $\mathbf{K}$ & $\mathrm{Ca}$ & Mg & Mn & $\mathbf{F e}$ & $\mathbf{Z n}$ & $\mathrm{Cu}$ \\
\hline & \multicolumn{9}{|c|}{ kg.ha' ${ }^{-1}$} \\
\hline Idiap FL 106-11 & $298 \mathrm{a}$ & $61 b$ & $360 \mathrm{a}$ & $159 b$ & $16 \mathrm{~b}$ & $6,7 \mathrm{a}$ & $4,8 b$ & $1,4 \mathrm{a}$ & $0,4 a$ \\
\hline \multirow[t]{2}{*}{ Idiap FL 137-11 } & $196 \mathrm{~b}$ & $69 a$ & $213 b$ & $173 a$ & $21 \mathrm{a}$ & $6,1 b$ & $6,0 \mathrm{a}$ & $1,2 b$ & $0,3 b$ \\
\hline & \multicolumn{9}{|c|}{ kg.t ${ }^{-1}$ grano } \\
\hline Idiap FL 106-11 & $49 a$ & $10 \mathrm{a}$ & $59 a$ & $26 b$ & $2,7 \mathrm{~b}$ & $1,1 \mathrm{a}$ & $0,8 \mathrm{a}$ & $0,2 \mathrm{a}$ & $0,1 \mathrm{a}$ \\
\hline Idiap FL 137-11 & $32 b$ & $11 \mathrm{a}$ & $35 \mathrm{~b}$ & $28 \mathrm{a}$ & $3,5 \mathrm{a}$ & $1,1 \mathrm{a}$ & $0,9 \mathrm{a}$ & $0,2 \mathrm{a}$ & $0,1 \mathrm{a}$ \\
\hline
\end{tabular}

Letras diferentes en las columnas indican diferencias altamente significativas $(\mathrm{p}<0,001)$ / Different letters in the same columns indicates highly significant difference $(\mathrm{p}<0.001)$.

Cuadro 4. Ecuaciones de regresión y coeficiente de determinación para la extracción de nutrientes en dos variedades de arroz. Campo Experimental Pacífico Marciaga, Penonomé, Panamá. 2016.

Table 4. Regression equations and coefficient of determination for the nutrients extraction in two rice varieties. Pacifico Marciaga Experimental Field, Penonome, Panama. 2016.

\begin{tabular}{ccccc}
\hline \multirow{2}{*}{ Nutriente } & \multicolumn{2}{c}{ IDIAP FL 106-11 } & \multicolumn{2}{c}{ IDIAP FL 137-11 } \\
\cline { 2 - 5 } & \multicolumn{1}{c}{ Ecuación } & $\mathbf{R}^{2}$ & Ecuación & $\mathbf{R}^{\mathbf{2}}$ \\
\hline $\mathrm{N}=0,0658 \mathrm{x}^{1,8964}$ & $0,91^{* *}$ & $\mathrm{y}=0,0513 \mathrm{x}^{1,8669}$ & $0,75^{* *}$ \\
$\mathrm{P}$ & $\mathrm{y}=0,0016 \mathrm{x}^{2,3454}$ & $0,97^{* *}$ & $\mathrm{y}=0,0026 \mathrm{x}^{2,2538}$ & $0,97^{* *}$ \\
$\mathrm{~K}$ & $\mathrm{y}=0,0692 \mathrm{x}^{1,9273}$ & $0,87^{* *}$ & $\mathrm{y}=0,0608 \mathrm{x}^{1,3452}$ & $0,71^{* *}$ \\
$\mathrm{Ca}$ & $\mathrm{y}=0,0019 \mathrm{x}^{2,506}$ & $0,98^{* *}$ & $\mathrm{y}=0,0003 \mathrm{x}^{3,0231}$ & $0,93^{* *}$ \\
$\mathrm{Mg}$ & $\mathrm{y}=0,0027 \mathrm{x}^{1,8837}$ & $0,82^{* *}$ & $\mathrm{y}=2 \mathrm{E}-06 \mathrm{x}^{3,5294}$ & $0,96^{* *}$ \\
$\mathrm{Mn}$ & $\mathrm{y}=6 \mathrm{E}-05 \mathrm{x}^{2,5355}$ & $0,98^{* * *}$ & $\mathrm{y}=2 \mathrm{E}-05 \mathrm{x}^{2,8176}$ & $0,95^{* *}$ \\
$\mathrm{Fe}$ & $\mathrm{y}=1 \mathrm{E}-04 \mathrm{x}^{2,3645}$ & $0,98^{* * *}$ & $\mathrm{y}=6 \mathrm{E}-05 \mathrm{x}^{2,4876}$ & $0,90^{* *}$ \\
$\mathrm{Zn}$ & $\mathrm{y}=2 \mathrm{E}-05 \mathrm{x}^{2,531}$ & $0,91^{* * *}$ & $\mathrm{y}=6 \mathrm{E}-06 \mathrm{x}^{2,7408}$ & $0,92^{* *}$ \\
$\mathrm{Cu}$ & $\mathrm{y}=5 \mathrm{E}-06 \mathrm{x}^{2,4677}$ & $0,98^{* *}$ & $\mathrm{y}=0,0002 \mathrm{x}^{1,5835}$ & $0,88^{* *}$ \\
\hline
\end{tabular}

$* *=$ diferencia altamente significativa $(\mathrm{p}<0,001) / * *=$ highly significant difference $(\mathrm{p}<0.001)$.

germinado (ddg), y coincidió con las fases de embuchamiento (hinchamiento de la panícula dentro de la vaina de la hoja bandera antes de su emergencia) y floración, la IDIAP FL 137-11 presentó dos picos de absorción que coincidieron con el máximo macollamiento (45 ddg) y el embuchamiento (Figura 3).

\section{Curva de absorción de fósforo}

Las variedades de arroz presentaron una dinámica de absorción de $\mathrm{P}$ diferenciada $(\mathrm{p}=0,005)$; IDIAP FL 137-11 presentó una absorción máxima entre el primordio floral y el embuchamiento (60 a 75 ddg) y en la etapa 


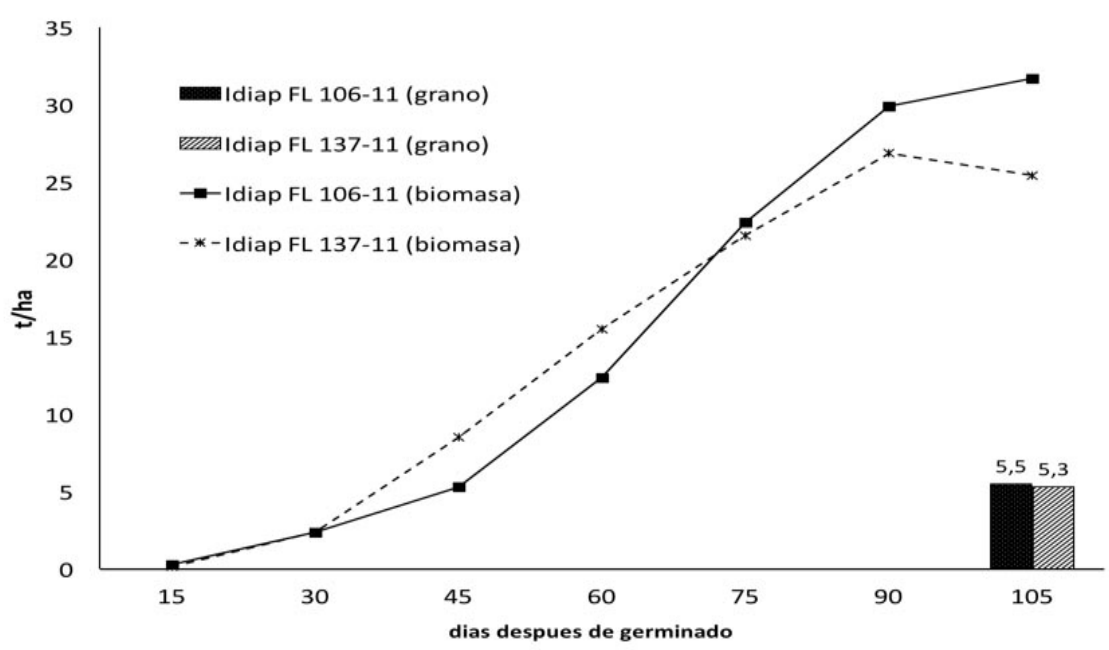

Figura 2. Producción de biomasa y rendimiento de grano para las variedades de arroz IDIAP FL 106-11 e IDIAP FL 137-11. Campo Experimental Pacífico Marciaga, Penonomé, Panamá. 2016.

Figure 2. Biomass production and grain yield for rice varieties IDIAP FL 106-11 and IDIAP FL 137-11. Pacifico Marciaga Experimental Field, Penonome, Panama. 2016.

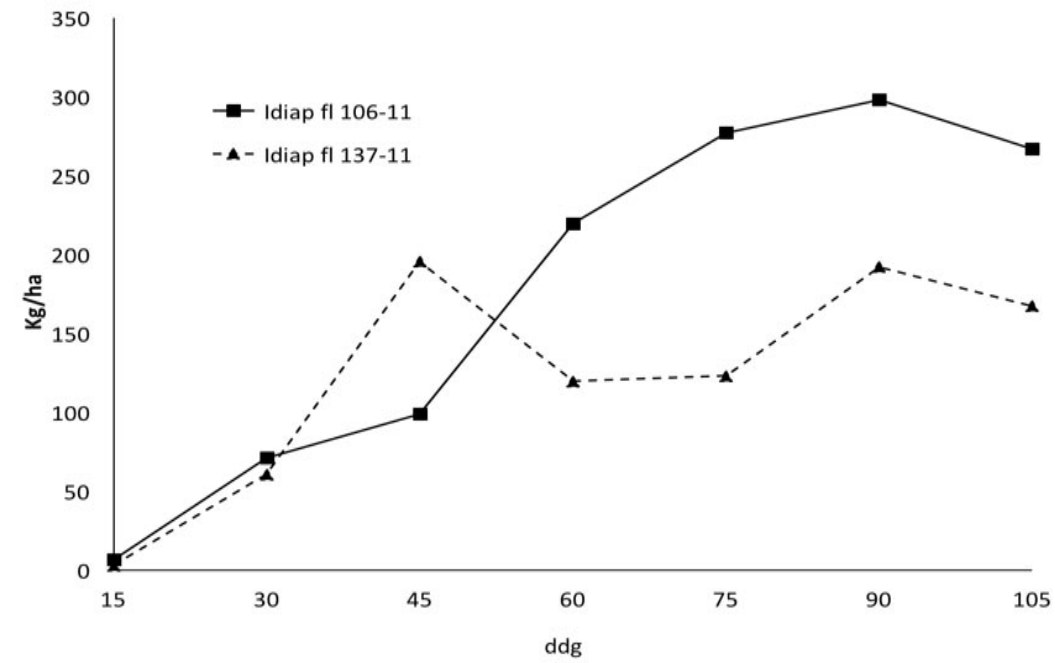

Figura 3. Absorción de nitrógeno de acuerdo con la variedad de arroz (IDIAP FL 106-11 e IDIAP FL 137-11) y su etapa fenológica. Campo Experimental Pacífico Marciaga, Penonomé, Panamá. 2016.

Figure 3. Nitrogen absorption according to the rice variety (IDIAP FL 106-11 and IDIAP FL 137-11) and its phenological stage. Pacifico Marciaga Experimental Field, Penonome, Panama. 2016.

de maduración (105 ddg), mientras que, la IDIAP FL 137-11 presentó su máxima extracción entre las etapas fenológicas comprendidas entre el primordio floral y la floración (Figura 4). 


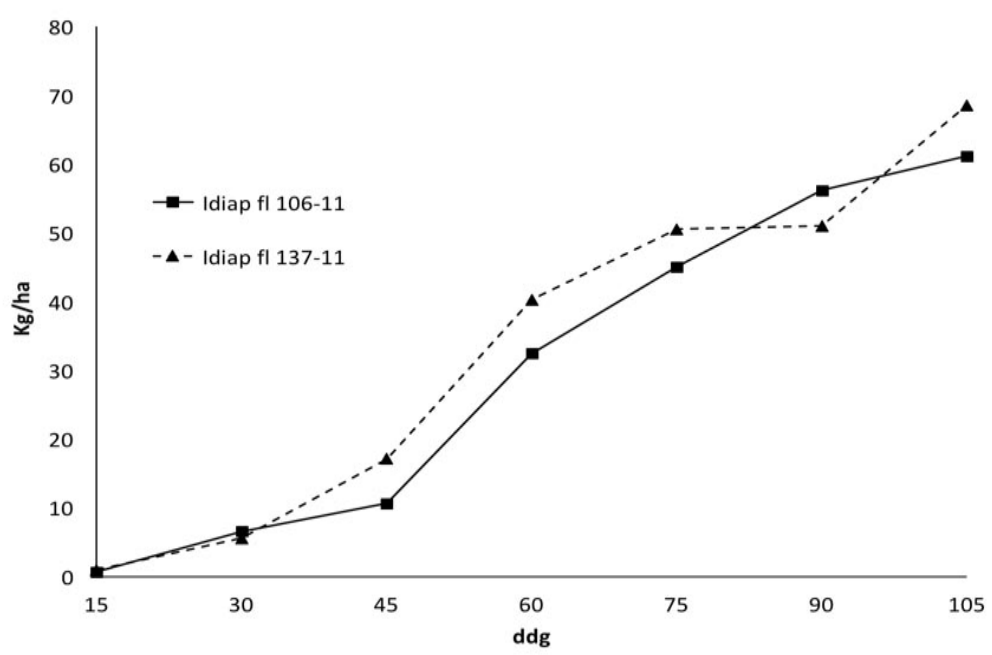

Figura 4. Absorción de fósforo de acuerdo con la variedad de arroz (IDIAP FL 106-11 e IDIAP FL 137-11) y su etapa fenológica. Campo Experimental Pacífico Marciaga, Penonomé, Panamá. 2016.

Figure 4. Phosphorus absorption according to the rice variety (IDIAP FL 106-11 and IDIAP FL 137-11) and its phenological stage. Pacifico Marciaga Experimental Field, Penonome, Panama. 2016.

\section{Curva de absorción de potasio}

La absorción de $\mathrm{K}$ tuvo un comportamiento distinto en ambas variedades $(\mathrm{p}<0,001)$, la IDIAP FL 106-11 mostró un incremento en la absorción a inicio de macollamiento (30 ddg) y luego presentó su máxima extración de los 75 a 90 ddg, lo que coincidió con las fases de embuchamiento y floración. La IDIAP FL 137-11 presentó dos picos de absorción que coinciden con el máximo macollamiento y la etapa de floración (Figura 5).

\section{Curva de absorción de calcio}

La tendencia en la absorción de Ca por ambas variedades fue similar $(\mathrm{p}=0,130)$, con un incremento continuo en la absorción a medida que se desarrolló la planta, con una diferencia al llegar a la fase de floración, donde la IDIAP FL 137-11 detuvo su extracción y la IDIAP 106-11 continuó absorbiendo Ca hasta el final de su ciclo fenológico (Figura 6).

\section{Curva de absorción de magnesio}

El Mg fue absorbido por ambas variedades de manera distinta ( $\mathrm{p}=0,003)$, la IDIAP FL 137-11 mostró una gran absorción durante el embuchamiento y luego en la etapa de maduración del cultivo, y la IDIAP FL 106-11 mostró un pico de absorción entre las fases de inicio del primordio floral y el embuchamiento o panzoneo (Figura 7).

\section{Curva de absorción de manganeso}

La dinámica en absorción de Mn presentó un comportamiento similar en ambas variedades ( $\mathrm{p}=0,096)$, con un pico de máxima extracción bien marcado durante el periodo de floración (Figura 8). 


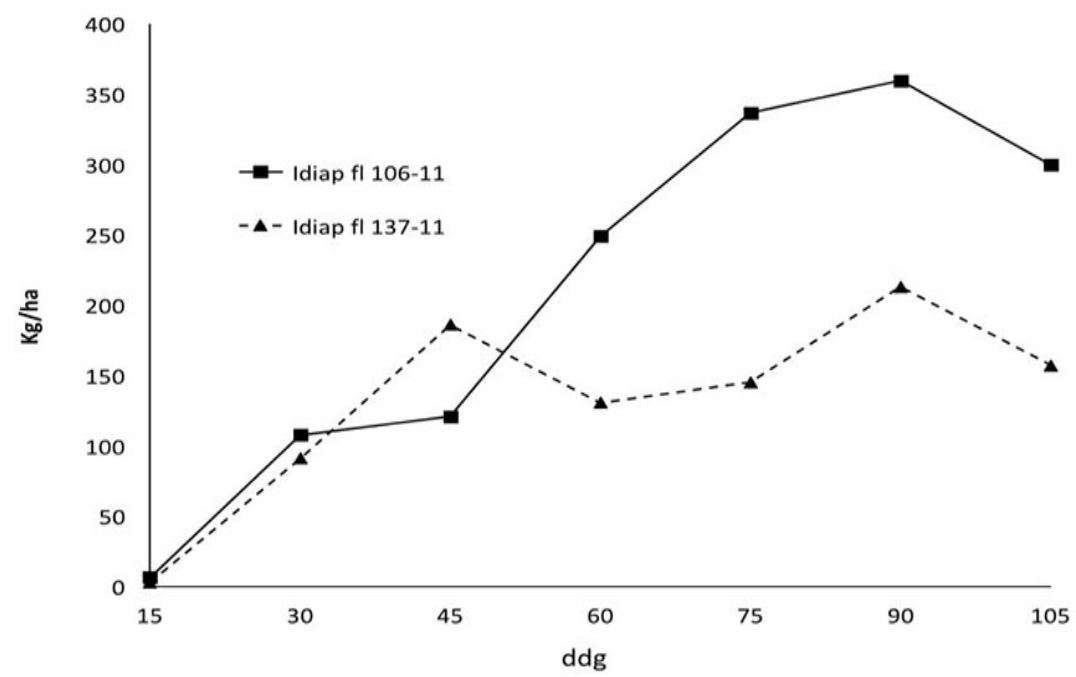

Figura 5. Absorción de potasio de acuerdo con la variedad de arroz (IDIAP FL 106-11 e IDIAP FL 137-11) y su etapa fenológica. Campo Experimental Pacífico Marciaga, Penonomé, Panamá. 2016.

Figure 5. Potassium absorption according to the rice variety (IDIAP FL 106-11 and IDIAP FL 137-11) and its phenological stage. Pacifico Marciaga Experimental Field, Penonome, Panama. 2016.

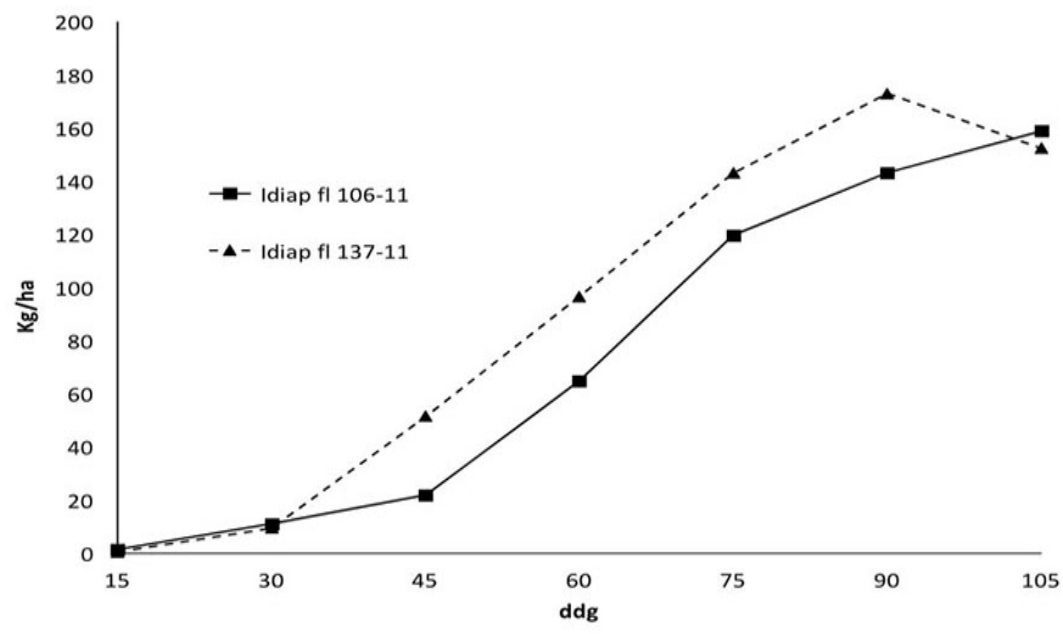

Figura 6. Absorción de calcio de acuerdo con la variedad de arroz (IDIAP FL 106-11 e IDIAP FL 137-11) y su etapa fenológica. Campo Experimental Pacífico Marciaga, Penonomé, Panamá. 2016.

Figure 6. Calcium absorption according to the rice variety (IDIAP FL 106-11 and IDIAP FL 137-11) and its phenological stage. Pacifico Marciaga Experimental Field, Penonome, Panama. 2016. 


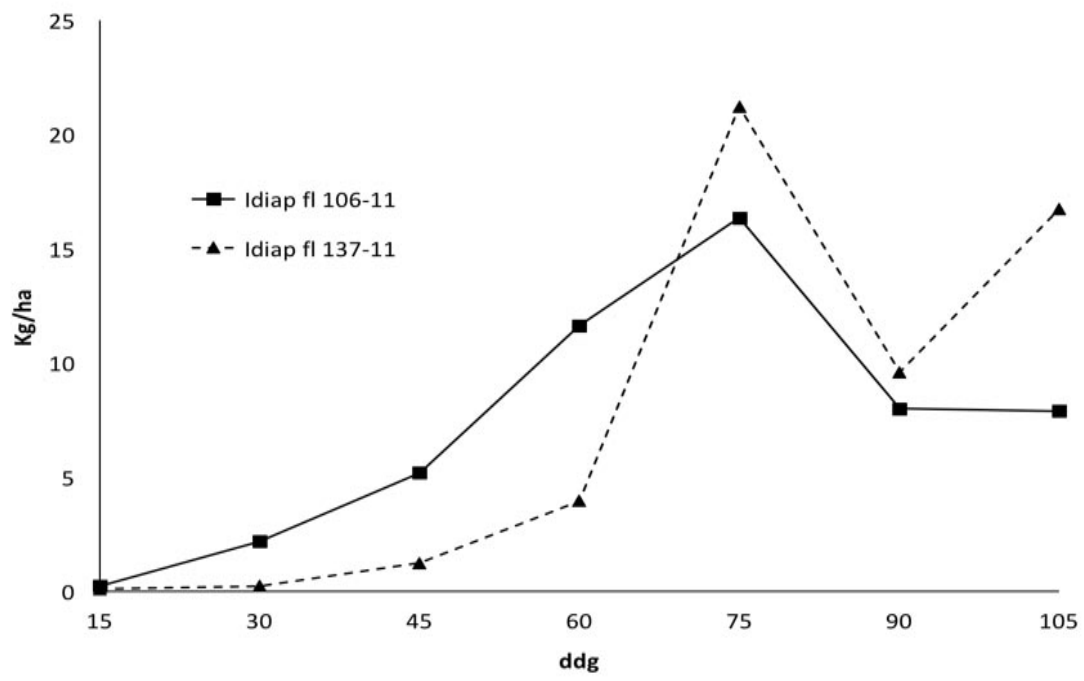

Figura 7. Absorción de magnesio de acuerdo con la variedad de arroz (IDIAP FL 106-11 e IDIAP FL 137-11) y su etapa fenológica. Campo Experimental Pacífico Marciaga, Penonomé, Panamá. 2016.

Figure 7. Magnesium absorption according to the rice variety (IDIAP FL 106-11 and IDIAP FL 137-11) and its phenological stage. Pacifico Marciaga Experimental Field, Penonome, Panama. 2016.

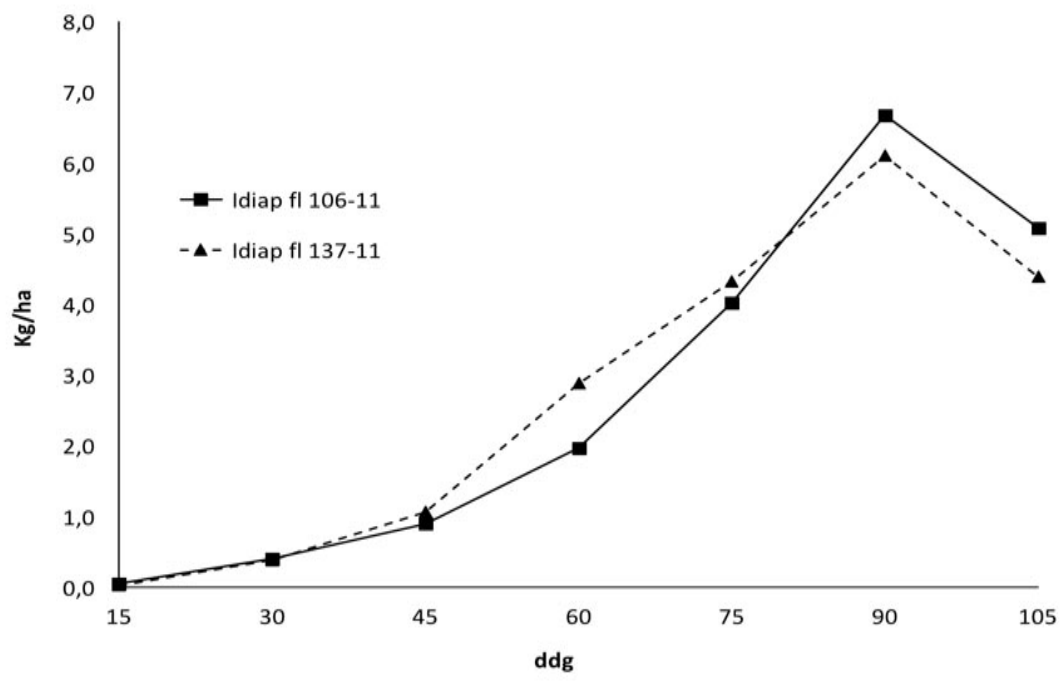

Figura 8. Absorción de manganeso de acuerdo con la variedad de arroz (IDIAP FL 106-11 e IDIAP FL 137-11) y su etapa fenológica. Campo Experimental Pacífico Marciaga, Penonomé, Panamá. 2016.

Figure 8. Manganese absorption according to the rice variety (IDIAP FL 106-11 and IDIAP FL 137-11) and its phenological stage. Pacifico Marciaga Experimental Feld, Penonome, Panama. 2016. 


\section{Curva de absorción de hierro}

La dinámica de absorción de Fe por ambas variedades fue distinta $(\mathrm{p}<0,001)$, donde la IDIAP FL 137-11 mostró un incremento en el máximo macollamiento y luego una extracción máxima durante la floración, mientras que la IDIAP FL 106-11 tuvo la máxima extracción durante la floración (Figura 9).

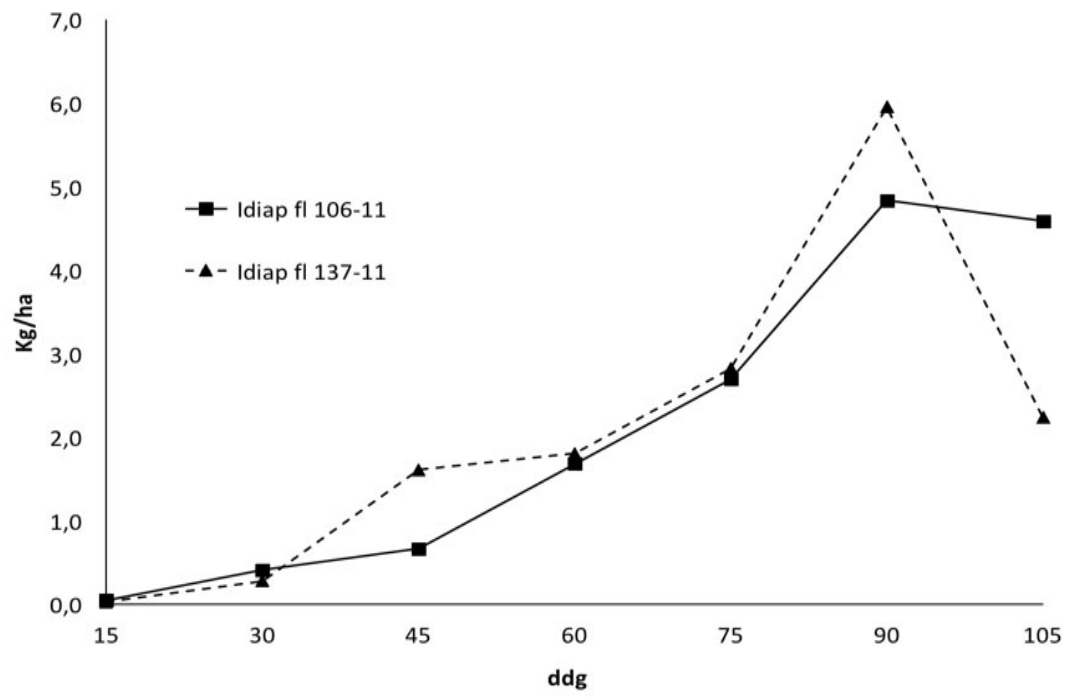

Figura 9. Extracción de hierro de acuerdo con la variedad de arroz (IDIAP FL 106-11 e IDIAP FL 137-11) y su etapa fenológica. Campo Experimental Pacífico Marciaga, Penonomé, Panamá. 2016.

Figure 9. Iron extraction according to the rice variety (IDIAP FL 106-11 and IDIAP FL 137-11) and its phenological stage. Pacifico Marciaga Experimental Field, Penonome, Panama. 2016.

\section{Curva de absorción de zinc}

La dinámica de absorción de Zn difirió con la variedad ( $\mathrm{p}=0,034)$, en cuanto la IDIAP 137-11 presentó un incremento en el máximo macollamiento y su máxima extracción durante la etapa fenológica de floración, mientras que la IDIAP FL 106-11 tuvo un pico de absorción durante el inicio del macollamiento y su mayor absorción se encontró en la fase de embuchamiento (Figura 10).

\section{Curva de absorción de cobre}

Las dos variedades de arroz mostraron un comportamiento diferente en la extracción de $\mathrm{Cu}$ durante su fenología ( $\mathrm{p}=0$,021), donde la IDIAP FL 106-11 tuvo una máxima extracción entre las fases de embuchamiento y floración, y la IDIAP FL 137-11 incrementó su absorción durante el máximo macollamiento y luego presenta un pico de absorción durante la floración (Figura 11). 


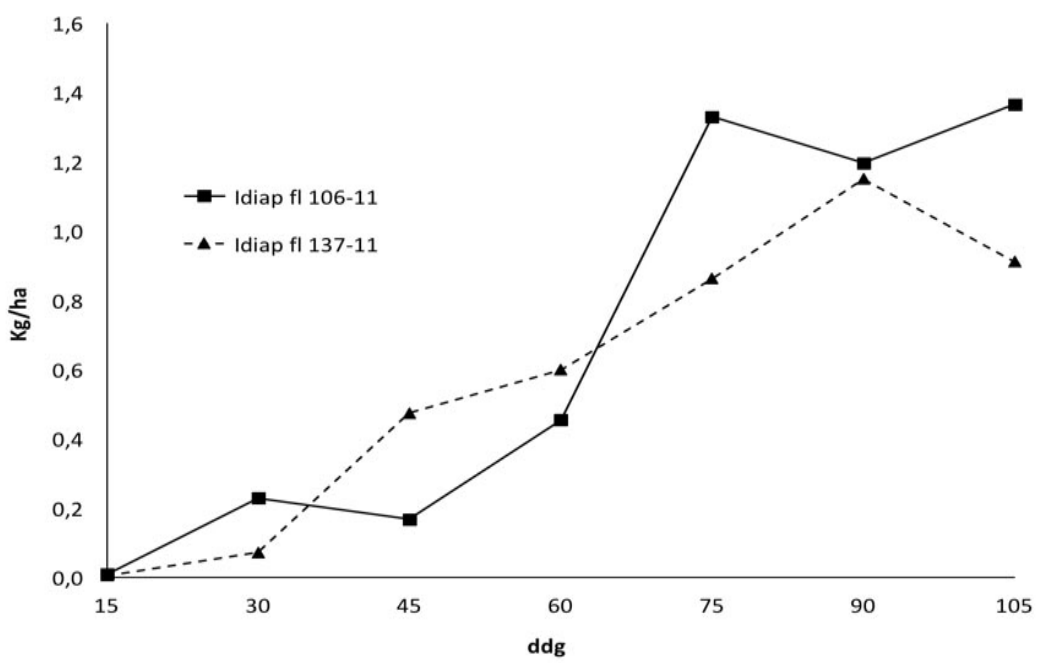

Figura 10. Absorción de zinc de acuerdo con la variedad de arroz (IDIAP FL 106-11 e IDIAP FL 137-11) y su etapa fenológica. Campo Experimental Pacífico Marciaga, Penonomé, Panamá. 2016.

Figure 10. Zinc absorption according to the rice variety (IDIAP FL 106-11 and IDIAP FL 137-11) and its phenological stage. Pacfico Marciaga Experimental Field, Penonomé, Panamá. 2016.

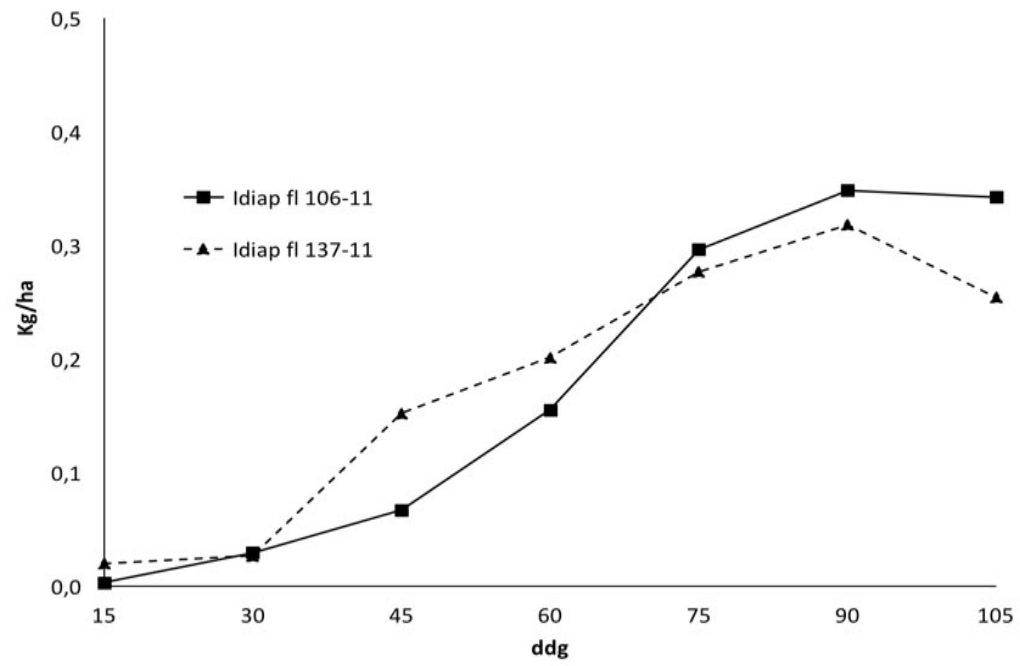

Figura 11. Absorción de cobre de acuerdo con la variedad de arroz (IDIAP FL 106-11 e IDIAP FL 137-11) y su etapa fenológica. Campo Experimental Pacífico Marciaga, Penonomé, Panamá. 2016.

Figure 11. Copper absorption according to the rice variety (IDIAP FL 106-11 and IDIAP FL 137-11) and its phenological stage. Pacífico Marciaga Experimental Field, Penonomé, Panamá. 2016.

\section{Orden de importancia de nutrientes según cantidades absorbidas}

El orden de importancia de los nutrientes nutrientes para ambas variedades de arroz y con base en la cantidad total extraída durante todo el ciclo fenológico, fue de $\mathrm{K}>\mathrm{N}>\mathrm{Ca}>\mathrm{P}>\mathrm{Mg}>\mathrm{Fe}>\mathrm{Zn}>\mathrm{Mn}>\mathrm{Cu}$ (Figura 12). 


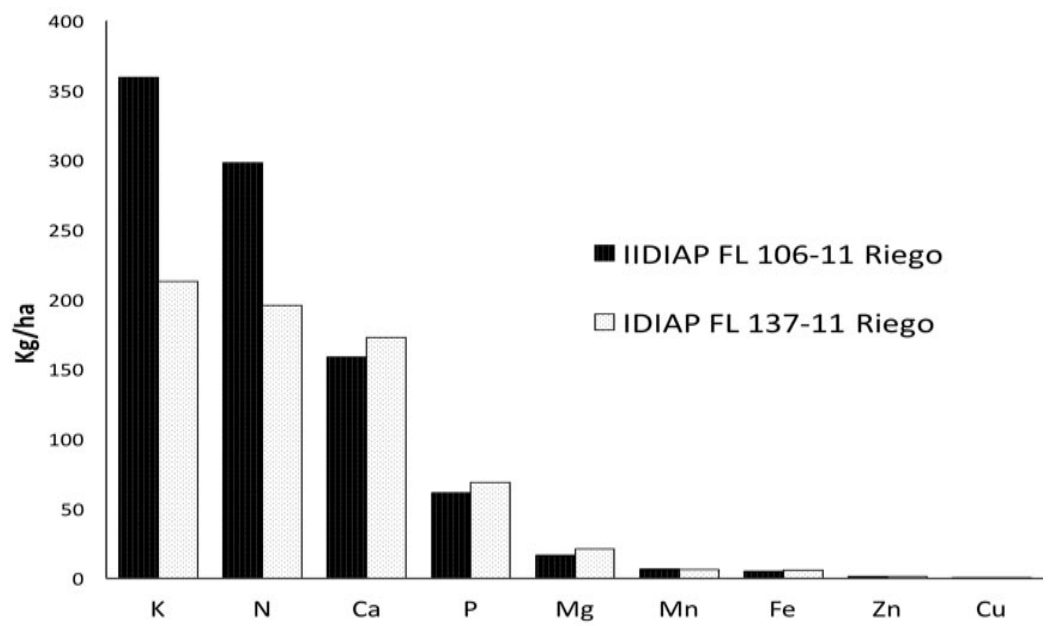

Figura 12. Orden de importancia de nutrientes según las cantidades absorbidas por las variedades de arroz IDIAP FL 106-11 e IDIAP FL 137-11. Campo Experimental Pacífico Marciaga, Penonomé, Panamá. 2016.

Figure 12. Nutrients order of importance according to quantities absorbed by the varieties of rice IDIAP FL 106-11 and IDIAP FL 137-11. Pacífico Marciaga Experimental Field, Penonomé, Panamá. 2016.

\section{Extracción de nutrientes por distintas secciones de la planta de arroz}

Al observar la absorción de nutrientes de forma relativa (Figura 13), se distingue que en la raíz, el nutriente que se acumuló en mayor proporción fue el Fe, con casi un $80 \%$ del total, mientras que el K y P concentraron menos del $5 \%$ de su total en este órgano (Figura 13). En los órganos foliares (hoja y tallo), fue donde se dio la mayor

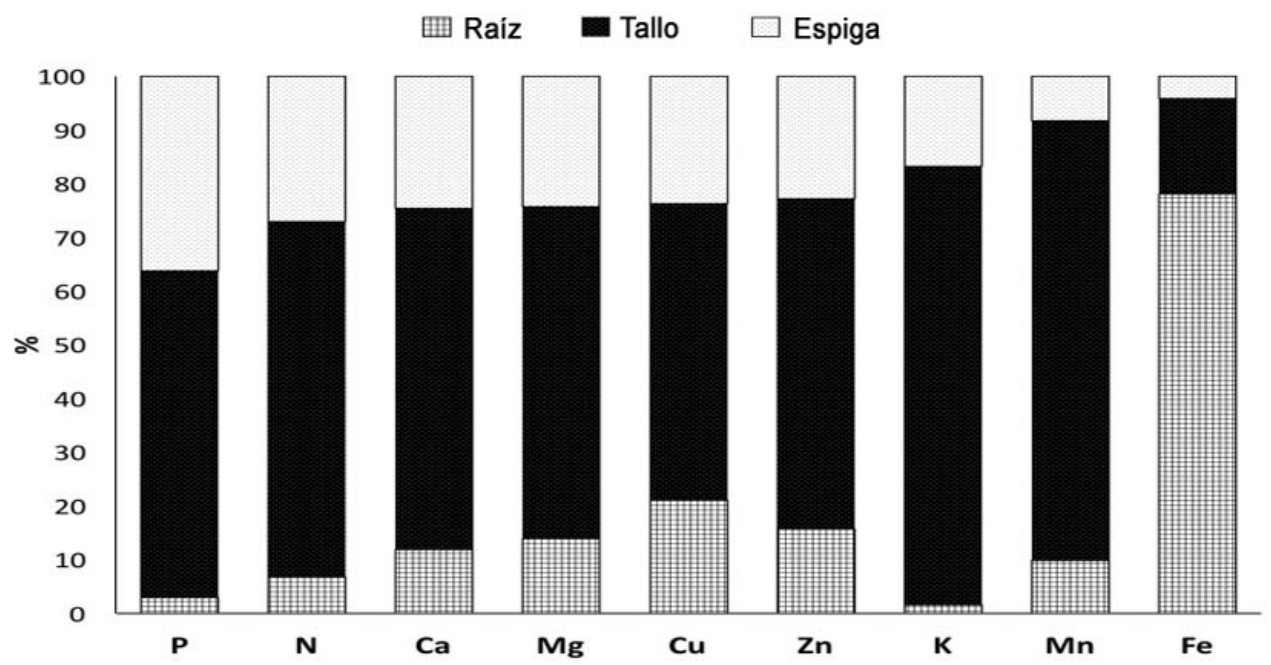

Figura 13. Absorción relativa de nutrientes por distintas secciones en dos variedades de arroz IDIAP FL 106-11 e IDIAP FL 137-11. Campo Experimental Pacífico Marciaga, Penonomé, Panamá. 2016.

Figure 13. Relative absorption of nutrients by different sections in two rice varieties IDIAP FL 106-11 and IDIAP FL 137-1. Pacífico Marciaga Experimental Field, Penonomé, Panamá. 2016. 
concentración de los nutrientes absorbidos por la planta, donde el K y Mn concentraron más del $80 \%$ de su total, y solo el Fe tuvo menos del $50 \%$ concentrado en esta parte de la planta de arroz. El nutriente que proporcionalmente destinó la mayor cantidad de su absorción por la planta hacia la espiga fue el P, con casi un $40 \%$, mientras que el Mn y el Fe concentraron menos del $10 \%$ de su total absorbido en esta sección.

\section{Discusión}

La variedad IDIAP FL 106-11 extrajo mayor cantidad de nutrientes que la variedad IDIAP FL 137-11, a pesar de que la productividad fue similar, lo que se traduce en una eficiencia más baja en cuanto a la partición de fotosintatos. La variedad IDIAP FL 137-11 necesitó menor cantidad de nutrientes para producir una tonelada de arroz que la IDIAP FL 106-11. La eficiencia también se puede determinar con el índice de cosecha (IC), el cual es la relación entre la biomasa seca producida y la producción de grano seco ( $0 \%$ humedad), donde la IDIAP FL 137-11 presentó un IC de 0,35 y la IDIAP FL 106-11 de 0,31. Esto concuerda con lo expuesto por Bouman et al. (2014), respecto a que las variedades modernas tropicales de corta duración presentan un IC que va de 0,3 a 0,5 en la estación lluviosa con alta nubosidad.

La ecuación de tipo potencial fue la que mejor se ajustó a la tendencia de los datos, y explicó mejor la correlación entre la edad de cultivo y la extracción de nutrientes en ambas variedades.

Los resultados de este estudio coinciden con los reportes de Molina y Rodríguez (2012), Amador y Bernal (2012), quienes encontraron la máxima extracción de $\mathrm{N}$ en la etapa de máximo macollamiento mientras que Solarzano (2003), reportó el máximo macollamiento y el inicio de floración como las etapas de mayor extracción de N; Name y Villarreal (2004) reportaron la etapa de llenado de grano como la de mayor extracción de $\mathrm{N}$ en el cultivo de arroz. El nitrógeno requiere un manejo cuidadoso, debido a que es muy susceptible a pérdidas por volatilización, lixiviación, desnitrificación, erosión y escorrentía, lo cual implica realizar un fraccionamiento momentos antes de que ocurran las etapas críticas de absorción, como inicio de macollamiento, máximo macollamiento y la diferenciación floral, para que el mismo este disponible en el suelo para su absorción por la planta.

A partir del inicio de la formación del primordio y hasta la floración del arroz, la planta absorbió el 58 \% del P total requerido (Molina y Rodríguez, 2012). Los resultados de esta investigación mostraron a las etapas reproductivas como las de mayor absorción de $\mathrm{P}$, coincidiendo con otros autores que afirmaron que las etapas de mayor demanda de fósforo son el macollamiento, grano maduro (Amador y Bernal, 2012) y la etapa de llenado de grano (Name y Villarreal, 2004). El P en el suelo presentó una baja movilidad, debido a que es adsorbido por las arcillas, lo que implicó una lenta disponibilidad, por lo cual las aplicaciones al momento de la siembra favorecen a que esté disponible en la etapa reproductiva que es la de mayor demanda, ya que tiene gran movilidad dentro de la planta.

Los resultados de la variedad IDIAP FL 106-11 coincidieron con los encontrados por Molina y Rodríguez (2012), quienes reportaron una absorción tardía muy alta durante la fase de maduración, que correspondió al $45 \%$ del K total absorbido; un resultado similar encontró Vargas (2002) donde más del $80 \%$ del K fue absorbido en la fase reproductiva y de maduración; mientras que, Name y Villarreal (2004) reportaron la etapa de llenado de grano como la de mayor extracción de K. Los resultados de la variedad IDIAP FL 137-11 se asemejaron a los de Amador y Bernal (2012), quienes reportaron la mayor absorción de K en la etapa de macollamiento. La dinámica del K en el suelo fue mas estable que la del N, donde no hay pérdidas por volatilización ni lixiviación, por lo cual muchos planes de fertilización de arroz (como el actual) realizan una sola aplicación al momento de la siembra, sin embargo, los resultados obtenidos sugieren que hay que hacer un fraccionamiento del mismo junto al fraccionamiento del $\mathrm{N}$.

Los resultados de absorción de Ca de este estudio difirieron de los trabajos realizados por Amador y Bernal (2012), quienes reportaron que las etapas de mayor demanda fueron macollamiento y floración; mientras que, 
Molina y Rodríguez (2012) identifican los estados de macollamiento y llenado de grano como las de mayor demanda de $\mathrm{Ca}$, pero en las variedades estudiadas en esta investigación, la mayor extracción se presentó en las etapas reproductivas del cultivo, con baja extracción en las etapas de macollamiento.

El Ca fue el tercer elemento más absorbido por el cultivo de arroz, sin embargo, este no forma parte de los planes de fertilización actuales, por lo cual se debería incluir, principalmente en suelos con bajo contenido del mismo.

En cuanto al Mg, los resultados de este estudio coincidieron con los encontrados por Amador y Bernal (2012), quienes reportan la etapa de floración como una de las de mayor demanda de este elemento.

En la absorción de Mn, los resultados de este experimento difirieron de los trabajos realizados por Amador y Bernal (2012), donde reportaron su mayor absorción en la etapa de macollamiento; sin embargo, fueron similares a los reportados por Molina y Rodríguez (2012), quienes ubicaron las etapas tardías como las de mayor extracción de Mn en el cultivo de arroz.

Trabajos realizados por Molina y Rodríguez (2012) y Amador y Bernal (2012), ubicaron la etapa de maduración como la de mayor extracción de Fe, lo cual difirió con esta investigación, ya que la etapa de floración fue la de mayor extracción de $\mathrm{Fe}$ en ambas variedades.

Los resultados mostraron las etapas reproductivas como las de mayor absorción de $\mathrm{Zn}$, coincidiendo con Molina y Rodríguez (2012) quienes reportaron que la mayor parte del zinc se absorbió durante la fase reproductiva; además, de Amador y Bernal (2012) identificaron la etapa de maduración como la de mayor extracción de este elemento.

Autores afirman que la mayor parte del cobre es absorbido durante la fase reproductiva (Molina y Rodríguez, 2012), pero otros encontraron que la etapa de maduración es la de mayor extracción de cobre (Amador y Bernal, 2012), ambos supuestos coinciden con los resultados de este estudio, donde las etapas reproductivas fueron las de mayor absorción de $\mathrm{Cu}$ en ambas variedades.

El orden de extracción de nutrientes para las variedades IDIAP 106-11 e IDIAP 137-11 mostró al K como el elemento extraído en mayor cantidad, seguido del N, Ca, P y Mg. Estos resultados coincidieron con los reportados por Molina y Rodríguez (2012), quienes encontraron el mismo orden de absorción por el cultivo de arroz; además Herber y Kraemer (2010) encontraron resultados similares, solo difirieron al reportar que el Mg se extrajo en mayor cantidad que el P. Los resultados obtenidos en esta investigación sugieren la incorporación a los programas de fertilización actuales nutrientes como $\mathrm{Ca}, \mathrm{Mg}, \mathrm{Cu}, \mathrm{Fe}$ y $\mathrm{Zn}$, principalmente en suelos con bajos contenidos de estos elementos.

La proporción en la cual los distintos órganos de la planta de arroz extrajeron los nutrientes es de gran relevancia en los programas de fertilización, en especial en aquellas zonas donde se cultiva de manera continua a través de los años, determinando así la cantidad de nutrientes que salen del sistema con la cosecha (grano) y los nutrientes que se reciclan a través de la reincorporación de los residuos vegetales al suelo una vez que estos se descomponen (raíz, tallo y hojas).

Los resultados de este estudio coincidieron con los reportados por Vargas (2002), Bertsch (2009) y los de Herber y Kraemer (2010), donde el P concentró cerca del $40 \%$ de su total en el grano, siendo el macronutriente que, proporcionalmente a su absorción, salió en mayor cantidad del sistema con la cosecha; mientras que, el K que se extrajo en mayor cantidad por el cultivo de arroz, se concentró en más del 80 \% en los órganos foliares (hoja y tallo), por lo cual podría reciclarse en el sistema con la reincorporación de los residuos del cultivo al suelo. Estos resultados se deben tener en consideración para ajustar los planes de fertilización actuales, tomando en cuenta los requerimientos de las variedades, la disponibilidad de los nutrientes en el suelo y la eficiencia de los fertilizantes a utilizar, se deben aplicar como mínimo, las cantidades de los nutrientes que son extraidos del suelo en la cosecha, para mantener la fertilidad del mismo. 


\section{Conclusiones}

Las dos variedades de arroz presentaron diferente extracción de nutrientes bajo las mismas condiciones, por lo cual se sugiere ajustar los planes de fertilización ( $120 \mathrm{~kg} \mathrm{~N} \cdot \mathrm{ha}^{-1}, 80 \mathrm{~kg} \mathrm{P}_{2} \mathrm{O}_{5} \cdot \mathrm{ha}^{-1}$ y $\left.80 \mathrm{~kg} \mathrm{~K}_{2} \mathrm{O} \cdot \mathrm{ha}^{-1}\right)$ para el cultivo de arroz recomendados actualmente (generalizada), con cada variedad.

La variedad IDIAP FL 137-11 presentó mayor eficiencia en la utilización de los nutrientes absorbidos con respecto al rendimiento de grano y al índice de cosecha. Esto implica que hay que hacer ajustes en los planes de fertilización actuales de acuerdo con la variedad que se utilice.

\section{Literatura citada}

Amador, J.C., e I.E. Bernal. 2012. Curva de absorción de nutrientes del cultivo de arroz (Oryza sativa L.) variedad Venezuela 21, en un suelo vertisol bajo condiciones del valle de Sébaco, Nicaragua. Tesis Lic., El Zamorano, Tegucigalpa, HND.

Baligar, V., and N. Fageria. 2007. Nutrient use efficiency in acid soil: Nutrient management and plant use efficiency. In: A.C. Moniz et al., editors, Plant-Soil interactions at low pH. Brazilian Soil Science Society, Viçosa, BRA. p. 75-95.

Bertsch. F. 2005. Estudios de absorción de nutrientes como apoyo a las recomendaciones de fertilización. Info. Agron. 57:1-10.

Bertsch. F. 2009. Absorción de nutrientes por los cultivos. Asociación Costarricense de la Ciencia del Suelo, San José, CRI.

Bouman, B., S. Haefele, G. Izzi, S. Peng, y T. Hsiao. 2014. Arroz: respuesta del rendimiento de los cultivos al agua. FAO, Roma, ITA.

Camargo, I., M.E. Quiros, y B. Zachrisson. 2014. Innovación tecnológica para el manejo integrado del cultivo de arroz en Panamá. Instituto de Investigación Agropecuaria de Panamá (IDIAP), Ciudad de Panamá, PAN.

Gutiérrez, H., y S.R. De-La-Vara. 2013. Análisis y diseño de experimentos. $3^{\text {ra }}$ ed. McGraw-Hill, México D.F., MEX.

Herber, L., y A. Kraemer. 2010. Curvas de absorción de nutrientes para cinco variedades de arroz de uso actual en Argentina. Instituto Nacional de Tecnología Agropecuaria, Corrientes, ARG.

Holdridge, L. 1967. Ecología basada en zonas de vidas. IICA, San José, CRI.

INPOFOS (Instituto de la Potasa y el Fósforo) 2004. Requerimientos nutricionales de los cultivos. Archivo Agronómico No 3. International Plant Nutrition Institute (IPNI), GA, USA. http://lacs.ipni.net/ipniweb/region/lacs.nsf/0/0B4CDA48FABB6 66503257967007DD076/\$FILE/AA\%203.pdf (consultado 9 ago. 2017).

Jaramillo, S. 1991. Pedones de campo y estaciones experimentales del IDIAP. Boletín Técnico. Instituto de Investigación Agropecuaria de Panamá, PAN.

Jarma, A., V. Degiovanny, y R. Montoya. 2010. Índices fisiotécnicos, fases de crecimiento y etapas de desarrollo de la planta de arroz. En: V. Degiovanny et al., editores, Producción eco-eficiente del arroz en América Latina. Tomo I. CIAT, Cali, COL. p. 60-82.

Magnifico, V., V. Lattazio, and G. Sarli. 1979. Growth and nutrient removal by broccoli. J. Am. Soc. Hort. Sci. 104(2):201-203.

Marschner, H. 1995. Mineral nutrition of higher plants. $2^{\text {nd }}$ ed. Academic Press, London, GBR.

Mejía, S., y J. Menjivar. 2010. Nutrición mineral del arroz. En: V.M. Beltramo et al., editores, Producción eco-eficiente del arroz en América Latina. Tomo I. CIAT, FLAR, y UNICORDOBA, Cali, COL. p. 306-335. 
Molina, E., y J.H. Rodríguez. 2012. Fertilización con N, P, K Y S, y curvas de absorción de nutrientes en arroz var. CFX 18 en Guanacaste. Agron. Costarricense 36(1):39-51. doi:10.15517/RAC.V36I1.9963

Name, B., y A. Cordero. 1987. Alternativas para uso y manejo de suelos ácidos en Panamá. En: S. Jaramillo editor, Compendio de los resultados de Investigación presentados en la II Jornada Científica. IDIAP, Santiago, PAN. p. 23.

Name, B., y J. Villarreal. 2004. Compendio de resultados de investigación del programa de suelos del IDIAP: Estudio de suelos ultisoles y alfisoles realizados en las Estaciones Experimentales de Calabacito, Guarumal y Río Hato. Instituto de Investigación Agropecuaria de Panamá, Ciudad de Panamá, PAN.

Perdomo, M.A., J. González, Y.C. De-Galvis, E. García, y O. Arregocés. 1985. Los macronutrimentos en la nutrición de la planta de arroz. En: J. Tascón et al., editores, Arroz: investigación y producción. CIAT, y PNUD, Cali, COL. p. 103-132.

Quirós-Mclntire, E., I. Camargo-Buitrago, J. Mejía, y C. Moreno. 2011. Descripción varietal de las líneas avanzadas en mejoramiento genético de arroz, IDIAP FL106-11 e IDIAP FL137-11. Instituto de Investigación Agropecuaria de Panamá, Penonomé, PAN.

Ramírez, F., y F. Bertsch. 1998. Metodología para generar curvas de absorción: presentación para curso. Universidad de Costa Rica, San José, CRI.

Ramírez, F., y F. Bertsch. 2002. Absorción de nutrientes por los frutos y bandolas de café caturra durante un ciclo de desarrollo y maduración de frutos en Aquiares, Turrialba, Costa Rica. Agron. Costarricense 26(1):33-42.

Ramírez, F., C. Henríquez, y F. Bertsch. 2000. Elaboración de las curvas de absorción de nutrientes y optimización del programa de fertilización para los cultivos Aster Blanco, Spring Aster, Solidazo y Solidaster. Informe final. Universidad de Costa Rica, San José, CRI.

Rodríguez, M., y V. Fórez. 2004. Elementos esenciales y beneficiosos. Universidad Nacional de Colombia, Bogota, COL.

Sancho, H. 1999. Curvas de absorción de nutrientes: Importancia y uso en los programas de fertilización. Inf. Agron. $36: 11$-13.

Solarzano, R. 2003. Crecimiento y nutrición del arroz (Oryza sativa L.) en Venezuela. Inf. Agron. 51:1-5.

Vargas, M. 2002. Fertilización con cuatro niveles de nitrógeno, fósforo y potasio y curvas de absorción de la variedad Fedearroz 50 en condiciones de secano favorecido. CONARROZ, San José, CRI.

Villarreal, J., y B. Name. 1996. Técnicas analíticas del laboratorio de suelos. Instituto de Investigación Agropecuaria de Panamá, Herrera, PAN.

Villarreal-Barrera, A.M. 2018. Respuesta a cuatro niveles de nitrógeno y curvas de absorción de nutrimentos para la variedad de arroz UP80FL, bajo condiciones de secano en dos localidades. Tesis MSc., Universidad de Panamá, Los Santos, PAN.

Wilcox, F., y J. Rivera. 2010. Manual de análisis del laboratorio especializado de suelos y tejido foliar del centro regional universitario de Los Santos. Universidad de Panamá, Los Santos, PAN.

Yoshida, S. 1978. Tropical climate and its influence on rice. IRRI Research Paper Series 20. International Rice Research Institute (IRRI), Los Baños, PHL. 